\title{
Flexible and reconfigurable data sharing for smart grid functions
}

\author{
Carsten Krüger*, Jorge Velasquez, Davood Babazadeh and Sebastian Lehnhoff \\ From The 7th DACH+ Conference on Energy Informatics \\ Oldenburg, Germany. 11-12 October 2018
}

* Correspondence: Carsten. Krueger@offis.de

OFFIS e.V., 26121 Oldenburg,

Germany

\begin{abstract}
Electrical power systems are undergoing major transformations as the share of decentralized energy resources increases. One important aspect is that the system is required to cope and adapt to the fluctuating behavior of these elements in order to maintain a stable and reliable operation. Consequently, this new challenges call for novel approaches for control and protection devices, both new and already deployed in the field. One alternative is the possibility to have a reconfigurable architecture, which ensures a modular and flexible operation of the system depending on the situation at hand. With this in mind, the main challenge addressed here is the configuration of the intelligent devices and the distribution of the data to ensure a resilient operation of the electrical grid.
\end{abstract}

Keywords: Cyber-resilience, Power generation control, Flexible control architecture

\section{Introduction}

Resilience is one important requirement for modern electrical power systems. This comes to hand with the ever increasing penetration of decentralized renewable energy sources. Consequently, new and flexible control and protection strategies are required to adapt to the uncertainties that renewable energies might introduce in the current electrical power systems (Buechner et al. 2014). Nowadays, the current state of the devices deployed within substations are used for limited and predefined functionalities, thus a flexible architecture with re-usable hardware is at the moment not realized (Jansen et al. 2005; Winter et al. 2010). With this in mind, re-configurable control and protection devices will play an important role in an increased resilient and reliable management of modern electrical power systems. For this reason, in the project i-Protect (Buescher et al. 2014), such architecture is proposed and the overall concept is outlined. The main contribution has been the development of several protection functions and its deployment to off-the-shelf hardware to achieve a certain degree of modularity and flexibility as presented in (Kleemann et al. 2011; Kleemann and Rehtanz 2011). Taking this into account, the project $\mathrm{i}$-Automate has been extending the aforementioned concept with the use of a data model for the description of the configuration of the hardware in use (for both control and protection functions). The used data model is based on the Substation Configuration description Language (IEC

(c) The Author(s). 2018 Open Access This article is distributed under the terms of the Creative Commons Attribution 4.0 International License (http://creativecommons.org/licenses/by/4.0/), which permits unrestricted use, distribution, and reproduction in any medium, provided you give appropriate credit to the original author(s) and the source, provide a link to the Creative Commons license, and indicate if changes were made. 
$61850 \mathrm{SCL}$ ) but extended to not only include information about a particular substation but a complete description of the devices deployed across the electrical power system. However, in this research paper the main focus is the data distribution concept that enables not only the configuration of the intelligent devices but also the reliable communication among the distributed components.

\section{Concept}

To present the already motivated idea of a highly reconfigurable and reliable communication service in a conceptual process three separate aspects are investigated. First, an overview of the involved components and information are shown which are used in the context of the project i-Automate. Second, specific information for the used communication service is given. Finally, the specific information is used to present the concept for a highly reconfigurable data distribution service.

The project i-Automate is focused on the LV/MV grid and therefore Intelligent Electronic Devices (IEDs) are used to measure grid information. The IEDs are able to run different applications and functionalities like voltage control or state estimation. To configure the functionalities in a standardized way the IEC 61850 Substation Configuration description Language (SCL) file is used. For example, the IED Capability Description (ICD) file describes all capabilities of an IED. Based on this a specific Instantiated IED Description (IID) file can be processed to describe the functions and objects of a specific IED. For some of the specified functions it is necessary to have not only their own provided measurements but also information from other parts of the grid, which is provided by other IEDs. Therefore, the IEDs needs an application that provides a communication service and under the consideration of reliability and resilience, this service must be highly reconfigurable. In the context of i-Automate a middleware called OpenDDS is used. For a better comprehension, the next paragraph explains the capabilities of OpenDDS in a more detail.

OpenDDS is an open source C++ implementation of the Object Management Group (OMG) Data Distribution Service (DDS). DDS itself is a middleware standard and provides a publish-subscribe pattern to enable scalability, real-time, high-performance and interoperable data exchange. In the following, Fig. 1 introduces the data-centric publish-subscribe (DCPS) concept of OpenDDS.

- Domain: Is a fundamental partitioning unit and each of the other entities belongs to a domain and can only interact with other entities of the domain.

- Topic: Describes the interaction between the publishing and subscribing application, so that samples for different instances of topics are published and the subscribers requests on this instances of topics to get the data.

- DataWriter: The data writer's type-specific interface is used to publish samples of the topic and is responsible for serializing the data.

- Publisher: Disseminate the data to all relevant subscribers in the domain.

- Subscriber: It receives the data from publisher and passes it to the relevant data readers connected.

- DataReader: takes the data from the Subscriber, to deserialize it into the appropriate type of topic. Afterwards the data reader forward the information to the application. 


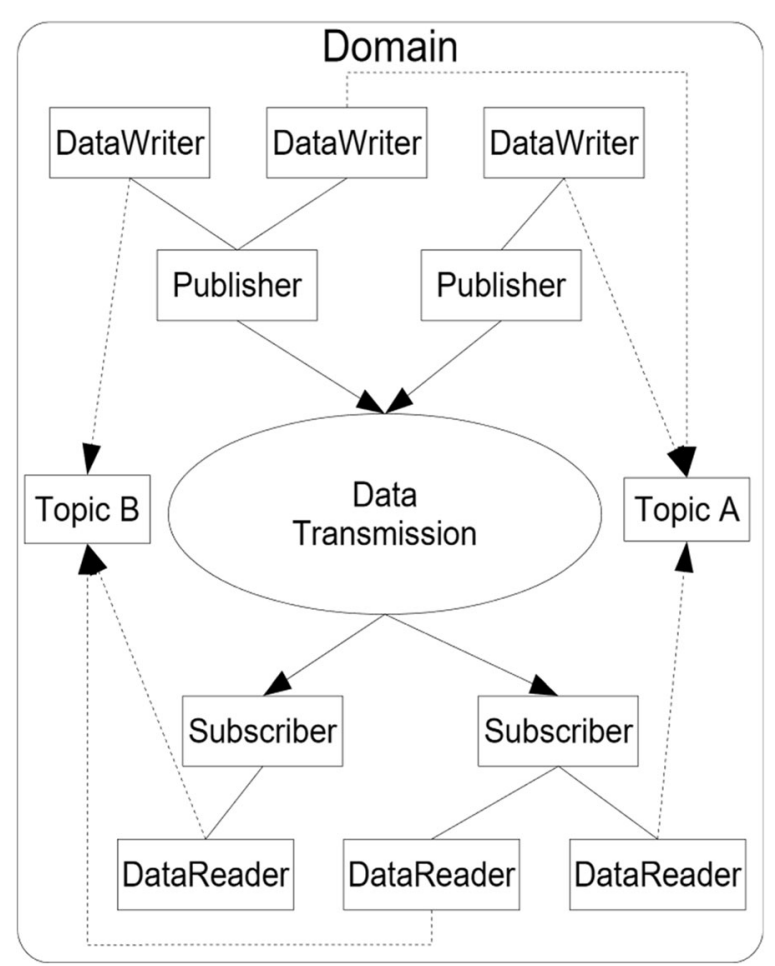

Fig. 1 DCPS conceptual overview (Computing O. Inc. n.d.)

For the data transmission, OpenDDS provide a specific extensible transport framework (ETF) as shown in Fig. 2. This framework gives the opportunity of different pluggable transport and discovery options. For this reason, TCP, UDP, multicast, shared-memory and RTPS_UDP are available for the transport and two types of discovery are distinguished. First, the centralized discovery with DCPSInfoRepo, which use a centralized repository style, that runs as a separate process allowing publishers and subscribers to discover one another centrally. On the other side, the RTPS discovery that use the peer-to-peer style and utilizes the RTPS protocol to advertise availability and location information.

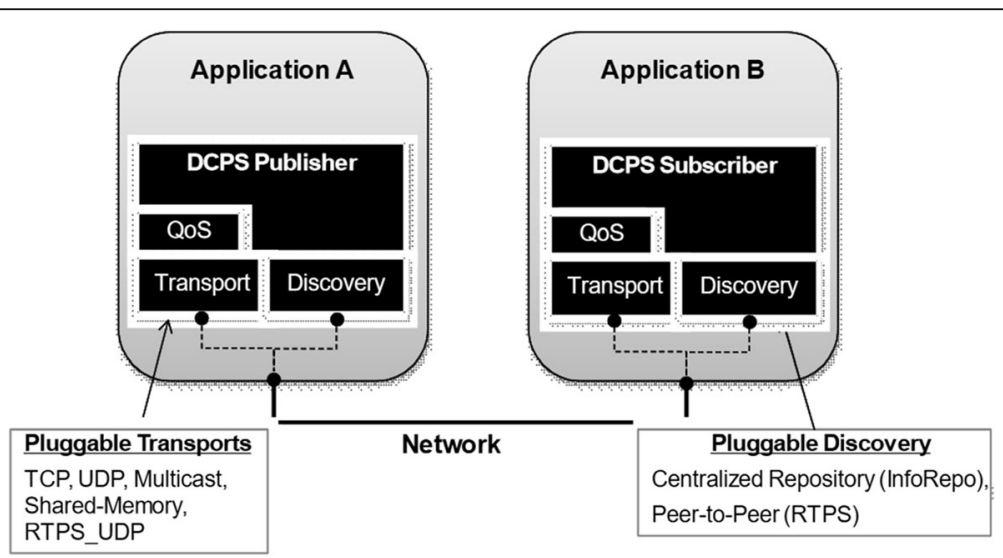

Fig. 2 OpenDDS extensible Transport Framework (Computing O. Inc. n.d.) 
Together with the information about the middleware that should be used as the data distribution service the conceptual proceeding can be described as follows. Consequently, based on the topologically separation of different IEDs it is possible to build cluster of IEDs there have a similar topological location (as presented in Fig. 3).

For each of the cluster one main responsible IED can aggregate or preprocess information depending on the functionality that is provided. In large scale application a subset of responsible IEDs of all clusters can build again a cluster and provide a hierarchical structure until one main responsible IED is able to aggregate all information. Each of the cluster map to one domain of the DCPS provided by OpenDDS and all IEDs inside of this domain can reach each other by multicast.

The data that have to be exchanged are modelled as a topic inside of each domain and each IED contains publisher and subscriber implementation together with their topic information of their interest. Now each IED inside of a cluster is able to publish or subscribe there samples. A concrete example can be seen by Fig. 4 where three IEDs are connected in the same domain and each of them provides a publisher and subscriber. In this example, the master IED wants to aggregate the information from topic 1 and topic 2 for that the device must only know the topics. The slaves 1 and 2 publish on three topics also only need to know about the topics and not about other IEDs. To illustrate why this concept of flexible and reconfigurable data sharing increase the reliability and resilience of the system, the following scenarios can be investigated:

- Based on Fig. 4 the master provide a function that needs the information of topic 1 and topic 2. In the case of disconnection of the master, one of the slaves can take over the task. Therefore, slave one reconfigure his communication links by

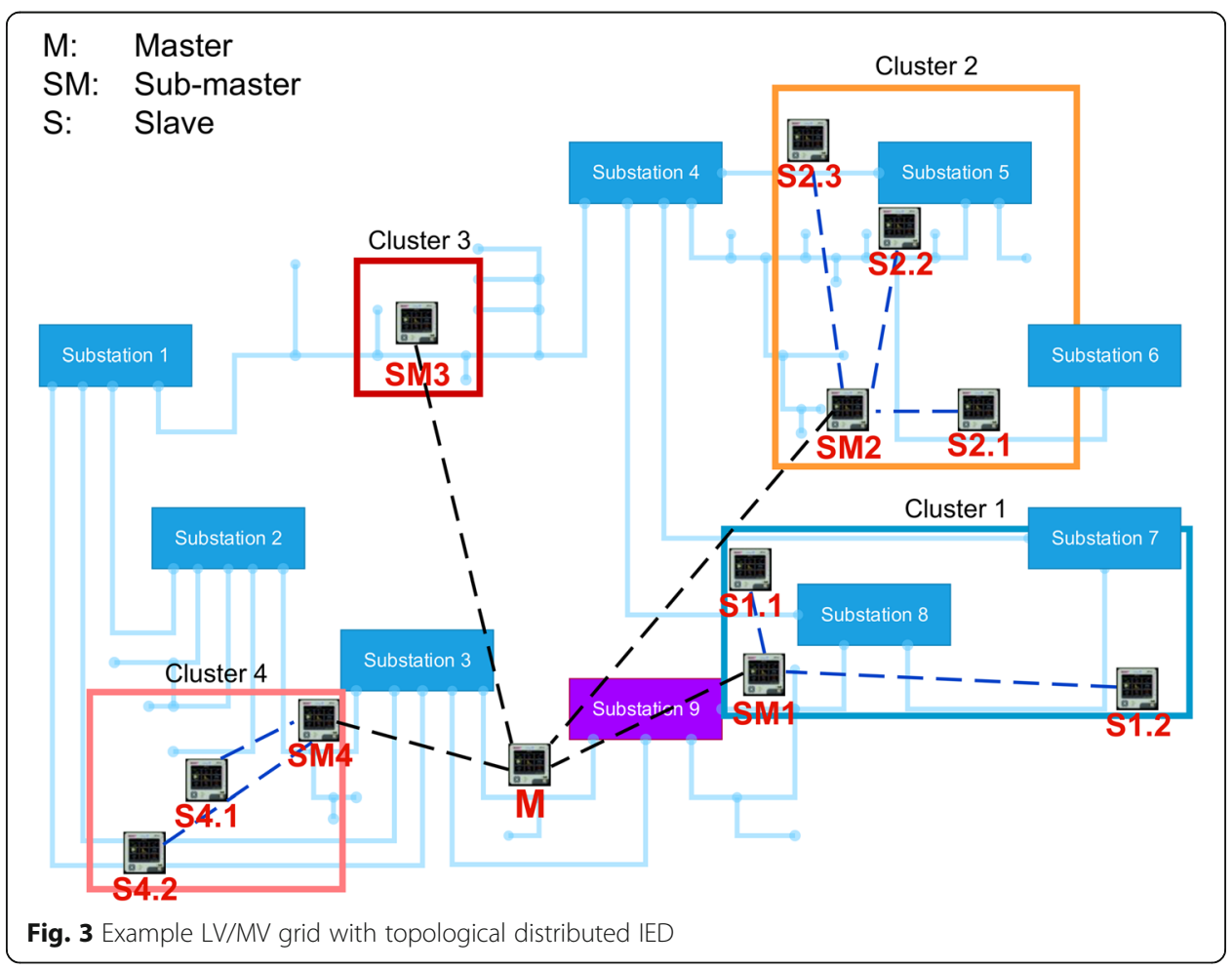




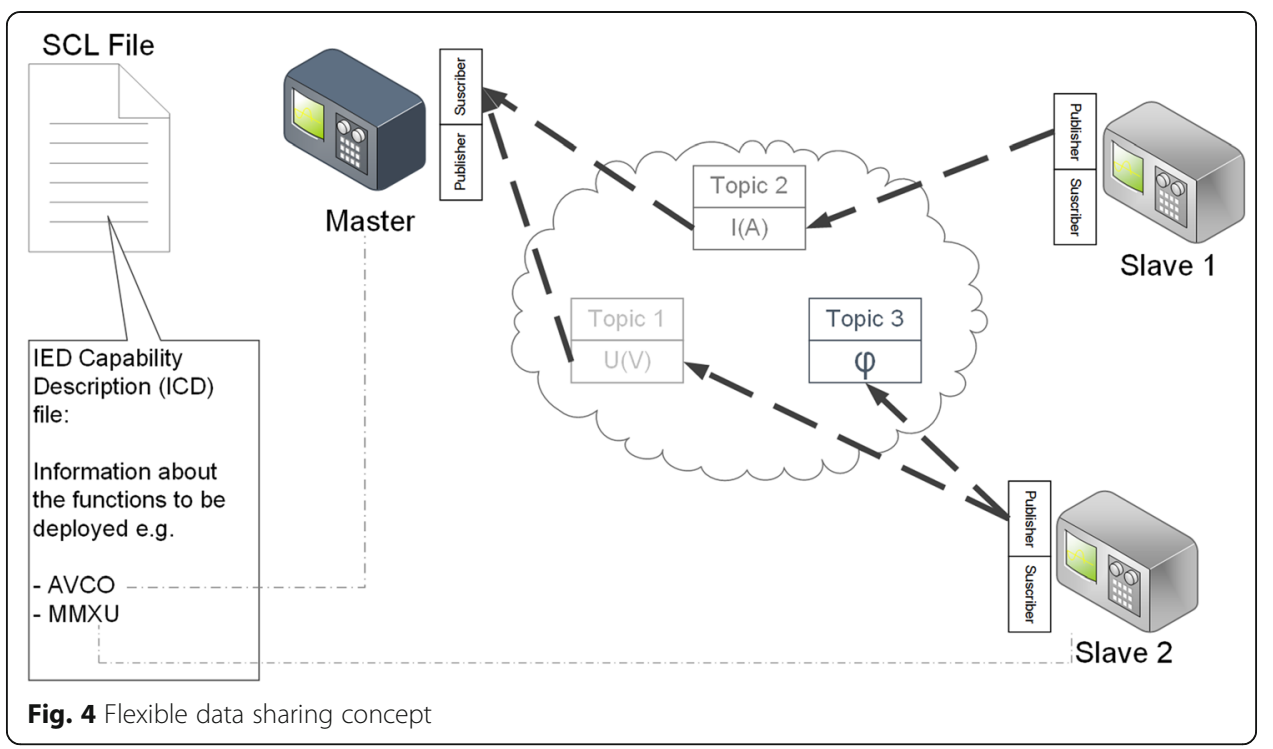

subscribing on the information of topic 1 . To realize this reconfiguration the slave one needs only the knowledge about the functionality that runs normally on the master and the necessary topics. To subscribe on the topic 1 OpenDDS use IP multi-cast to share meta-data about the provided topics.

- For the case that two IEDs are available in the same substations and both are able to provide the same data, OpenDDS provides a technique called hot-swap that can be used to switch the publisher for a topic in real time to avoid that parts of the grid information are unavailable.

To configure the OpenDDS on each IED an extension of the SCL file is been used. This extension provides an additional file with the information about the topics that each of the IEDs have to publish or subscribe. This file is used on a separated server where OpenDDS are preconfigured. With regard to the underlying architecture of the IEDs these configured configuration of OpenDDS will be cross compiled and transferred to the devices. Finally all publisher provides there topics and the data samples that should be exchanged and all subscriber subscribe on the topics of their interest.

\section{Conclusion and outlook}

In this research paper, the main concept of the project $\mathrm{i}$-automate is presented. One of the aims of this particular research is the decentralized communication of intelligent devices leading to an enhanced and more flexible control and protections architecture. Consequently, the main contribution of the project is to increase the reliability and resilience of the electrical grid when dealing with highly fluctuating renewable resources. Important to realize is that this contribution is at its early stages and more work is required towards the implementation of the OpenDDS framework in off-the-shelf hardware that is able to realize several control and protection functionalities, namely decentralized voltage control and state estimation. 


\section{Abbreviations}

DCPS: Data-centric Publish-subscribe; DDS: Data Distribution Service; ICD: IED Capability Description; IED: Intelligent Electronic Device; IID: Instantiated IED Description; RTPS: Real-time Publish-subscribe; SCL: Substation Configuration Language; TCP: Transmission Control Protocol; UDP: User Datagram Protocol

\section{Acknowledgements}

This research is conducted at OFFIS as part of the project "i-Automate" funded by Federal Ministry for Economic Affairs and Energy (BMWi).

\section{Funding}

Publication costs for this article were sponsored by the Federal Ministry for Economic Affairs and Energy (BMWi).

\section{Availability of data and materials}

Not applicable.

\section{About this supplement}

This article has been published as part of Energy Informatics Volume 1 Supplement 1, 2018: Proceedings of the 7th $\mathrm{DACH}+$ Conference on Energy Informatics. The full contents of the supplement are available online at https:// energyinformatics.springeropen.com/articles/supplements/volume-1-supplement-1.

\section{Authors' contributions}

SL and DB developed the concept and revised the manuscript. JV wrote the introduction and CK implemented and wrote the concept. All authors have read and approved the final manuscript.

\section{Competing interests}

The authors declare that they have no competing interests.

\section{Publisher's Note}

Springer Nature remains neutral with regard to jurisdictional claims in published maps and institutional affiliations.

Published: 10 October 2018

\section{References}

Buechner J, Katzfey J, Moser A, Schuster H, Dierkes S, van Leeuwen T, et al. (2014) Moderne Verteilernetze für Deutschland (Verteilernetzstudie). In: BMWi (ed) Studie im Auftrag des Bundesministeriums für Wirtschaft und Energie (BMWi), 1st edn, Bonn

Buescher M, Kube M, Lehnhoff S, Piech K, Rohjans S, Trefke J (2014) Towards a process for integrated IEC 61850 and OPC UA communication: using the example of smart grid protection equipment. IECON 2014 - 40th Annu Conf IEEE Ind Electron Soc, pp 3605-3611

Computing O. Inc. OpenDDS Developer's Guide. (n.d.). http://download.objectcomputing.com/OpenDDS/OpenDDS-latest.pdf. Accessed 10 Aug 2018

Jansen A, Molly J, Neddermann B, Bachmann U, Gerch H, Grebe E, et al. (2005) Energiewirtschaftliche Planung für die Netzintegration von Windenergie in Deutschland an Land und Offshore bis zum Jahr 2020. In: DENA (ed) denaNetzstudie I, 1st edn, Berlin

Kleemann M, Rehtanz C (2011) Centralized substation automation system based on off-the-shelf devices. 4th Eur Conf HV MV substation equip

Kleemann M, Rehtanz C, Nenning A (2011) Power system protection for distributed generators based on off the-shelf components. IET Conf Renew Power Gener RPG 2011

Winter W, Neddermann B, Knorr K, Grave K, Lindenberger D (2010) Integration erneuerbarer Energien in die deutsche Stromversorgung im Zeitraum 2015-2020 mit Ausblick auf 2025. In: DENA (ed) dena-Netzstudie II, 1st edn, Berlin

\section{Submit your manuscript to a SpringerOpen ${ }^{\circ}$ journal and benefit from:}

- Convenient online submission

- Rigorous peer review

- Open access: articles freely available online

High visibility within the field

- Retaining the copyright to your article

Submit your next manuscript at $\boldsymbol{\nabla}$ springeropen.com 